\title{
Erratum
}

\section{Synthesis and Structure of Fluorescent Chelate Boron Complexes of 4-Anilinomethylidene-1-benzazepine-2,5-dione Ligands}

Nadine Tolle, Ute Dunkel, Luciano Oehninger, Ingo Ott, Lutz Preu, Tobias Haase, Soenke Behrends,

Peter G. Jones, Frank Totzke, Christoph Schächtele, Michael H. G. Kubbutat, Conrad Kunick* Synthesis 2011, 2848.

In the caption to Figure 6, the scale was incorrectly given in units of micromolar. The correct scale is $20 \mu \mathrm{m}$. 